\title{
LA DIFÍCIL TAREA DE CLASIFICAR UNIVERSIDADES
}

\section{Rankings y clasificaciones como reacción a la heterogeneidad institucional}

Se suele decir que mientras más "grande" es una palabra, más difícil es definirla. Palabras grandes como "iglesia", "familia", "nación" o "justicia" carecen de definiciones ampliamente compartidas. Quizás la excepción más notable es la palabra "universidad". Todos estamos de acuerdo, matices más o menos, en lo que es una universidad, y si se nos pidiera dar un concepto de ella, diríamos algo así como que es una institución dedicada a crear, cultivar y enseñar conocimiento.

Sin embargo, este arquetipo cubre una cada vez más amplia variedad de formas concretas de expresión. Esta diversidad, mucho más marcada en algunos países que en otros ${ }^{1}$, mueve a usuarios, reguladores y analistas a procurar introducir alguna medida de orden en sistemas que tienden a confundir. Una herramienta para ordenar son los rankings. Otra, que discutiremos aquí, son las clasificaciones.

Clasificación (o tipología) y ranking no es lo mismo. El ranking busca ordenar de mejor a peor según un conjunto ponderado de indicadores de desempeño. Una clasificación, en cambio, busca agrupar las instituciones por tipos homogéneos o, al menos, por semejanza, a la luz de ciertos atributos significativos para definir el perfil y la naturaleza de la organización. Sin embargo, como en educación superior los atributos de clasificación suelen ser los mismos o muy parecidos a los que son de interés para confeccionar rankings, no es inusual que las clasificaciones sean usadas en éstos

1 Pensemos, por ejemplo, en los sistemas de educación superior de Estados Unidos, Filipinas, Rusia, China o Chile, como ejemplos de heterogeneidad, en comparación con los de Holanda, Canadá, Singapur o Nueva Zelanda, en el otro extremo. 
o se confundan con ellos. Este ha sido el sino de la mundialmente conocida clasificación de universidades, la de la Carnegie Foundation for the Advancement of Teaching ${ }^{2}$, de EE.UU. El problema afecta también a las asociaciones selectivas de universidades, como la Association of American Universities, que cuando fue fundada en 1900 no tenía el aura de club de las mejores universidades de EE.UU. y Canadá que tiene hoy. Las asociaciones más nuevas de esta clase, en cambio, como la League of European Research Universities (LERU) o la Innovative Research Universities, de Australia, buscan deliberadamente diferenciarse de la masa de universidades a través de la formación de un exclusivo club de instituciones.

En nuestro medio, las clasificaciones de universidades también son usadas como rankings, ya sea en forma deliberada o inadvertida. La categoría de universidad tradicional es un ejemplo, aunque cada vez menos confiable. El número de años de vigencia de la acreditación es otro, pese a que la Comisión Nacional de Acreditación (CNAP), agencia encargada del proceso, se ha cuidado de prevenir contra tal uso de la duración de este periodo. Esta desnaturalización de las clasificaciones y tipologías es esperable e inevitable en un mercado tan heterogéneo como el chileno (o el de EE.UU.) en que hay tanta necesidad de información que permita discriminar entre universidades y tan poca disponible oficialmente para uso general.

Para clasificar es necesario, primero, tener un punto de vista y, luego, al menos un criterio de clasificación. Uno puede, por ejemplo, tomar el punto de vista de un postulante a la universidad y clasificar a las universidades según prestigio, o por los puntajes mínimos de ingreso de la carrera de interés en diversas universidades, o según el costo del arancel (dentro de lo cual, la existencia del crédito fiscal es un criterio importante), o por la ubicación geográfica del campus, o por el perfil socioeconómico de los estudiantes, entre otros criterios.

2 Véase el sitio http://www.carnegiefoundation.org/classifications, 
Si se toma el punto de vista del gobierno, se puede elegir el criterio del acceso a subsidios directos del Estado y, entonces, se puede clasificar a las universidades en instituciones con aporte fiscal directo y sin este aporte; o se puede usar como criterio la naturaleza jurídica de la entidad y se tendrá como resultado dos clases: las universidades estatales y las privadas.

Se puede, en fin, considerar a las universidades por sus funciones y tomar como criterio, por ejemplo, el de la investigación o el de la docencia de posgrado; o mirarlas desde el punto de vista de la historia y usar como norma la fecha de fundación, como lo hace la actual legislación universitaria chilena. Hay aún muchas otras opciones de clasificación.

\section{El informe "Guiar el Mercado" como ejercicio de clasificación}

El ejercicio más importante y sistemático de clasificación intentado en Chile hasta ahora aparece en la sección final de la tercera parte de "Guiar el Mercado: Informe sobre la Educación Superior en Chile" (2005, pp. 209-220)3 , de José Joaquín Brunner, Gregory Elacqua, Anthony Tillett y colaboradores. Su tipología busca desarrollar una clasificación que responda a la heterogeneidad institucional y a las actuales condiciones de mercado en las universidades chilenas.

Las variables consideradas para la clasificación son la naturaleza jurídica de la universidad (pública o privada); el tamaño, medido por la matrícula total; la cobertura de áreas profesionales en que la institución ofrece carreras de pregrado; la selectividad, medida por el porcentaje de alumnos de altos puntajes en las pruebas de selección sobre la matrícula nueva de pregrado; la capacidad de investigación; la misión declarada por las instituciones; el mercado regional de pertenencia, y la posición de prestigio en los rankings.

3 Ver documento http://www.uai.cl/p4_home/site/asocfile/ASOCFILE120050121095042.pdt Los números de página del informe a los que se hace referencia aquí son los de esta versión electrónica del texto y no los del libro publicado a mediados de 2005. 
No obstante el amplio rango de variables consideradas, el informe propone como el patrón principal de clasificación al nivel de selectividad de las universidades. Adopta así el punto de vista del mercado de estudiantes de pregrado y el criterio fundamental de la tipología es función de las decisiones de matrícula de los alumnos con los mejores puntajes en las pruebas de selección universitaria.

Dicho informe discurre sobre la base de que no hay un mercado nacional de universidades (salvo, en alguna medida, para la Universidad de Chile y la Pontificia Universidad Católica de Chile), sino varios mercados o nichos, definidos principalmente por el tipo y tamaño de la institución, su localización geográfica, su oferta de carreras y su prestigio (p. 131-132). Para apoyar la hipótesis de la segmentación regional de los mercados se presenta, para cada mercado postulado (Norte, Litoral, Metropolitano, Centro Sur y Austral), la proporción de alumnos que provienen desde fuera del respectivo mercado regional, la que los autores consideran baja. Es decir, la poca movilidad extrarregional de los estudiantes sugeriría que los mercados son regionales. Dentro de cada región, postulan los autores del informe, "los mercados regionales universitarios se segmentan, básicamente, en términos de jerarquías selectivas; esto es, de la proporción de alumnos top y supertop que las instituciones son capaces de atraer (p. 166)".

Para hacer el análisis más manejable, los autores asumen que las decisiones de matrícula de los alumnos dependen del prestigio de la institución como un todo, aun cuando, en realidad, es más probable que esas decisiones respondan mejor a una estimación de la reputación de la carrera " $x$ " en la universidad " $y$ ". No hay que olvidar que los alumnos eligen primero la carrera y luego la institución. Los datos de puntajes por carrera de cada institución están disponibles en la base de datos "INDICES"^ que elabora el Consejo Superior de Educación (CSE), pero su análisis requeriría un enfoque estadístico más complejo que el de "Guiar el Mercado". Trabajar con instituciones en vez de hacerlo con carreras en instituciones es, entonces, una opción conveniente

4 Indicadores, Números y Datos sobre Instituciones y Carreras de Educación Superior. 
para reducir el volumen de datos que es necesario procesar, pero no se debe perder de vista la distorsión que encierra.

El énfasis en la selectividad de alumnos que caracteriza al referido informe se proyecta a la tipología de universidades que propone, pero en combinación con las otras variables antes señaladas (naturaleza pública o privada de la universidad, tamaño, cobertura disciplinaria de sus carreras, investigación, tipo de misión, mercado regional de pertenencia y ubicación en los rankings de prestigio). Este necesario enriquecimiento de los criterios de clasificación se ve menoscabado, sin embargo, por la ausencia de orden lógico para la aplicación de cada uno de ellos, razón por la cual, como se explicará, la clasificación pierde consistencia.

En efecto, si hay más de un criterio de clasificación se deben ordenar lógicamente, de manera de aplicar los más generales primero, es decir, lo que conforman las clases más comprensivas, para luego ir dividiendo las clases en grupos más pequeños conforme a criterios secundarios. Así, por ejemplo, si nos ubicamos en la perspectiva del gobierno y nos interesa la posición de la universidad frente a los mecanismos de aseguramiento de la calidad, uno podría primero clasificar a las universidades en autónomas y no autónomas y, luego, a las primeras en acreditadas y no acreditadas.

En la tipología propuesta por "Guiar el Mercado", en cambio, no se explica bien qué criterio o combinación de criterios determina en qué clase se incluye cada universidad. La selectividad debiera ser, según la intención declarada por los autores, el criterio primero y dominante para separar las categorías, pero no lo es, porque si bien en la categoría 1 están -como es de esperar, si el criterio principal es selectividad- las universidades con selectividad "muy alta", y en la categoría 3 las universidades son de selectividad "alta" la categoría 4 las universidades son de selectividad "media" 7 , en la

5 Universidades de Chile y Pontificia Católica de Chile.

6 Universidades Adolfo Ibáñez y de los Andes.

7 Universidades Tecnológica Metropolitana y Metropolitana de Ciencias de la Educación. 
categoría 2 hay juntas universidades de selectividad "alta" y "media". No hay, entonces, una progresión lógica de mayor a menor selectividad a lo largo de las primeras cuatro categorías.

Las variables "tamaño", "investigación", "prestigio" y "áreas del conocimiento" están aún más disgregadas. En la categoría 2, por ejemplo, hay universidades muy grandes, grandes y medianas; de media, alta y baja actividad científica; con cobertura completa, amplia y especializada, y con ubicaciones en los rankings de prestigio que van del lugar 3 al 15. El grupo 5 sería homogéneo si no fuese por la inclusión de una universidad de tamaño mediano-pequeño, concentrada y de muy baja investigación, en una categoría que reúne a universidades medianas, completas o amplias y de baja investigación ${ }^{9}$. Luego, la categoría de las universidades de selectividad media-baja -que debiera ser una sola si la selectividad fuese efectivamente el criterio dominante- está separada en dos grupos, según si son regionales (el grupo 5 antes comentado) o privadas ${ }^{10}$ (grupo 6). No se advierte la razón de la separación, especialmente si se considera que el grupo siguiente, el de baja selectividad ${ }^{11}$, reúne a universidades regionales y privadas en una sola categoría.

La nomenclatura de las categorías no ayuda a esclarecer las cosas. En las dos primeras categorías se habla de "liderazgo académico", en circunstancias que el liderazgo no es un criterio de clasificación (además de la dificultad para operacionalizarlo, si lo fuera), y la diferencia es que en categoría 1 el liderazgo es internacional

8 Selectividad alta: universidades Pontificia Católica de Valparaíso, de Santiago de Chile y de Talca. Selectividad media: universidades Austral de Chile, de Concepción, Técnica Federico Santa María y de La Frontera.

9 El grupo 5 está conformado por las universidades: Católica del Maule, Católica del Norte, de Antofagasta, de La Serena, de Valparaíso y del Bío-Bío.

10 Universidades: Alberto Hurtado, del Desarrollo, Diego Portales, Finis Terrae, Gabriela Mistral y Mayor.

11 Universidades: Arturo Prat, Católica de la Santísima Concepción, Católica de Temuco, de Atacama, de Los Lagos, de Magallanes, de Playa Ancha de Ciencias de la Educación, de Tarapacá, Academia de Humanismo Cristiano, Central de Chile, de Rancagua, del Pacífico, Iberoamericana de Ciencias y Tecnología, Andrés Bello, San Sebastián y Tecnológica Vicente Pérez Rosales. 
y en la 2 es nacional, introduciéndose nuevamente una dimensión -proyección nacional o internacional- ajena a los criterios de clasificación. Además, no se presenta la evidencia en que se apoya el alcance de esas proyecciones.

La categoría 3 ya no usa el descriptor "liderazgo académico" sino el de "liderazgo selectivo", con lo cual se alude, apropiadamente aquí -si dejamos pasar la ambigüedad de la expresión "liderazgo selectivo"-, al criterio base que supuestamente ordena la tipología. Pero en la categoría 4 se vuelve a desdibujar la nomenclatura y ya no se trata de liderazgos, sino de ubicación geográfica y cobertura disciplinaria: la categoría 4 se llama "metropolitanas especializadas" (era esperable el nombre "liderazgo metropolitano especializado", para mantener la línea). La categoría siguiente es clasificada según territorio y selectividad, y las que vienen después según naturaleza jurídica y selectividad.

Los textos que acompañan a la categorización reflejan las dificultades de los autores para encontrar elementos comunes entre las universidades que configuran la mayor parte de las categorías propuestas. Sin embargo, como solución, las explicaciones no ayudan mucho, toda vez que se introducen como descriptores juicios no respaldados por los datos que consigna el informe. De este modo, nos encontramos con universidades que tienen en común el ser "exitosas", que desempeñan una "función rectora a nivel nacional o local" y se destacan en la "irradiación cultural" (p. 212), mientras otras son descritas como dedicadas a la "formación de elites" (p. 213). Todo esto puede ser cierto, pero son atributos no documentados en el informe.

\section{Selectividad y formación de elites como criterios de clasificación}

La cuestión de la formación de elites merece una observación aparte, porque la facilidad con que algunas afirmaciones de "Guiar el Mercado" asocian selectividad con formación de líderes puede llevar 
a conclusiones que me parecen lejanas a la intención de los autores. Desde luego, salvo en el caso de las universidades de Chile y Pontificia Católica de Chile, no está acreditado que los líderes de la economía, las profesiones, la política o las artes provengan abrumadoramente de las universidades que reclutan a los alumnos de más altos puntajes. Ello es bien probable, me parece, pero no está estudiado. Tampoco sabemos si es efectivo que las universidades de la categoría 6 formen a los "mandos medios del cuadro directivo del país" (p. 216), que las de la categoría 7 preparen a los "mandos medios" (p. 218) -no directivos, suponemos en este caso, aunque no se explicita- o que las de la categoría 8 hagan "promoción social" (p. 220)12.

A la ausencia de evidencia que apoye estas afirmaciones, se suma la confusión entre lo que las universidades hacen, es decir, sus resultados, y lo que buscan hacer, esto es, sus objetivos. El que una universidad forme mandos medios no necesariamente va aparejado a una concepción de su misión como orientada a ese fin. Supongo que la mayoría de las universidades busca formar líderes, cualesquiera sean las características de entrada de sus alumnos.

Así las cosas, es dudoso el carácter diferenciador respecto de otras universidades que se atribuye a las misiones de las universidades de la categoría 3 (Adolfo Ibáñez y de los Andes), a saber: "por sus características y posicionamiento, ambas instituciones pertenecen a aquel grupo de universidades que buscan incidir en la formación de las elites culturales, empresariales y profesionales del país" (p. 213).

Además, en Chile, tanto el éxito en las pruebas de selección universitaria como las posiciones de liderazgo social están fuertemente asociados a la pertenencia del individuo a estratos socioeconómicos altos. Entonces, especialmente cuando se trata de universidades privadas selectivas y caras, que no disponen -a diferencia de las

12 Universidades: Adventista de Chile, Autónoma del Sur, Bernardo O'Higgins, Bolivariana, Católica Silva Henríquez, de Aconcagua, ARCIS, UNIACC, de Ciencias de la Informática, de Las Américas, de Viña del Mar, del Mar, Internacional SEK, La República, Mariano Egaña, Marítima de Chile, Miguel de Cervantes, de Puerto Varas y Santo Tomás. 
selectivas tradicionales- de crédito fiscal para sus potenciales alumnos pobres de altos puntajes, decir que una universidad altamente selectiva forma líderes tiene algo de razonamiento circular, en el sentido de que esas universidades trabajan con alumnos de estratos altos, que ingresan socialmente destinados al liderazgo y egresan a asumir sus destinos.

Esta cuestión remite a otra de carácter más general: la ausencia en "Guiar el Mercado" de la consideración de la clase social como factor de segmentación del mercado de la educación superior. Territorio y puntajes en la prueba de selección son, sin duda, importantes condicionamientos en las decisiones de los jóvenes que buscan ingresar a la universidad, pero también el precio de los estudios y el perfil socioeconómico de los alumnos con que se van a encontrar en la universidad que escojan. Este factor no necesita presentarse así de crudamente en la función de decisión del estudiante para ejercer su influencia: basta que se manifieste en forma indirecta, en las opciones que hacen los compañeros del colegio de cursos superiores, en las universidades cuyos nombres son conocidos para el alumno y sus padres, en los edificios universitarios que conocen porque están ubicados a lo largo de los recorridos urbanos que realizan, etc.

Volviendo ahora al análisis de los criterios de categorización, vale la pena detenerse un momento en la elección de indicador que hacen los autores del aludido informe para medir selectividad. Como se señaló, la variable de interés aquí es la de los puntajes en las pruebas de selección universitaria. Ahora bien, el indicador de puntajes puede construirse de dos maneras: como el porcentaje de los alumnos que ingresan a cada universidad que son de altos puntajes ("top" y "supertop" los llama el informe) o como el porcentaje de altos puntajes que ingresan a cada universidad. La distinción puede parecer trivial, pero no lo es. El primer indicador, que es el escogido por los autores, tiende a favorece a las universidades pequeñas y concentradas, porque el número de sus alumnos de buenos puntajes no se diluye entre muchos alumnos de menores puntajes, mientras que el segundo indicador tiende a favorecer a las universidades con 
más alumnos y más carreras, que tienden una red más amplia para conseguir buenos puntajes.

Así, por ejemplo, si se considera el porcentaje de alumnos matriculados en cada universidad que son de altos puntajes, en el primer lugar de selectividad se ubica la Pontificia Universidad Católica de Chile, pero si se utiliza el porcentaje de los alumnos de altos puntajes que ingresan a cada universidad, prima la Universidad de Chile. En el caso de las universidades privadas, si se usa el primer indicador, las más selectivas son: Adolfo Ibáñez, de los Andes, Diego Portales, Finis Terrae, Alberto Hurtado y del Desarrollo, todas ellas pequeñas, salvo la Diego Portales. Si, en cambio, se aplica el segundo indicador, las privadas más selectivas son: Diego Portales, Mayor, Andrés Bello, Adolfo Ibáñez y del Desarrollo, todas ellas grandes, salvo las dos últimas. Hay buenas razones para defender la opción por un indicador, como las hay para inclinarse por el otro. Lo importante del punto es tener presente que dependiendo de la opción que se haga respecto del indicador de selectividad, los resultados varían y, con ello, se afecta toda clasificación de universidades que se base en este criterio de selectividad.

\section{Clasificación según funciones}

Lo que nos lleva de vuelta al problema del punto de vista dominante de la tipología y a la pregunta ¿es la del mercado de estudios de pregrado la mejor perspectiva para clasificar universidades? Depende del uso que se le quiera dar a la clasificación. Si el usuario que se tiene en mente es el que participa en este mercado, entonces la elección parece razonable. Si el usuario objetivo es la comunidad de investigadores y analistas, como en el caso de la clasificación de instituciones de educación superior de la fundación Carnegie, esa perspectiva es menos relevante, y lo que interesa, más bien, son las funciones de las universidades como manifestación de sus misiones, o, dicho de otra forma, la misión según es encarnada en el comportamiento. En el caso de la clasificación Carnegie, referencia obligada aunque no sea sino porque se ha perfeccionado a lo largo de 30 años, el 
criterio principal de clasificación es el tipo de grado académico que la institución otorga, y como subcriterios (apropiadamente subordinados al anterior) la cantidad de graduados que cada institución produce y áreas disciplinarias cubiertas por ellos respecto de algunos grados académicos de especial interés, como los de magíster y doctor.

El desarrollo y evolución de la clasificación Carnegie entre 1973 y 2005 merecen una digresión aquí no sólo por su relativa antigüedad y enorme prestigio, sino también en relación con lo que se ha venido discutiendo en estas líneas, en cuanto ponen de relieve las dificultades y peligros inherentes a las clasificaciones en sistemas de educación superior que, como el de los EE.UU. o el de Chile, son altamente competitivos, y en los que, por lo tanto, la información de buena calidad está en alta demanda.

La clasificación Carnegie fue originalmente diseñada para asistir a la propia fundación en sus tareas, mediante la ordenación de la enorme heterogeneidad de la educación superior de EE.UU. en categorías de instituciones semejantes. Probó tener enorme utilidad para los investigadores de la educación superior, por cuanto permitía trabajar con segmentos de ésta cuyos marcos clasificatorios eran conocidos y respetados por la comunidad académica en general. La clasificación fue revisada en 1976, 1987, 1994, 2000 y 2005, para ajustarla a los cambios ocurridos en las instituciones y en las formas de uso de la tipología. Como señala la comisión Carnegie:

"Por más de tres décadas, la Clasificación Carnegie ha sido el marco principal para describir la diversidad institucional de la educación superior en los EE.UU. Ha sido ampliamente usada en el estudio de la educación superior, tanto para representar o controlar por diferencias institucionales, como en el diseño de estudios muestrales que representen adecuadamente a los grupos de instituciones, alumnos o profesores de interés". ${ }^{13}$

13 Ver sitio http://www.carnegiefoundation.org/classification. 
La comisión Carnegie lamenta que, no obstante sus continuas admoniciones, la clasificación es usada por postulantes, ex alumnos, empresas y académicos como un ranking de calidad, y por las propias instituciones como una escala de prestigio que pesa considerablemente en sus decisiones de desarrollo, en cuanto, en buena medida, ellas se orientan a "subir" al próximo escalón en la clasificación.

El fracaso de los esfuerzos recientes de la comisión para disminuir la gravitación de la clasificación (en cuanto ranking), por medio de disminuir en 2000 las categorías superiores de la clasificación, de cuatro -Investigación 1, Investigación 2, Doctorado 1 y Doctorado 2-a sólo dos categorías, ha llevado en la última revisión a la mucho más radical medida de reemplazar el sistema clasificatorio único por un conjunto de clasificaciones paralelas que tipifican a las instituciones una y otra vez desde distintas perspectivas. La tradición de la clasificación anterior, no obstante, se ha mantenido bajo el nombre de "clasificación básica", aunque con cambios respecto de la edición 2000.

Las nuevas clasificaciones, que se suman a la básica, se articulan sobre los siguientes criterios: características de la oferta de programas de pregrado, de la oferta de programas de postgrado, de los alumnos matriculados (subdividida en dos clasificaciones) y, finalmente, tamaño y carácter residencial de la institución.

La clasificación básica, por su parte, reconoce las siguientes clases:

- Universidades de doctorado, que gradúan al menos 20 doctores al año en programas académicos (es decir, no profesionales) y se subdividen en tres grupos según la magnitud en ellas de la función de investigación, medida por indicadores de gasto, personal de investigación y doctorados conferidos, tanto en total como por profesor.

- Universidades y colleges de master, que entregan al menos 50 grados académicos por año, divididos en tres subgrupos según la cantidad de dichos grados conferidos al año.

- Colleges de baccalaureate, que entregan diplomas de pregrado en 
programas de cuatro años y que no califican para las categorías anteriores, separados en tres grupos por especialidad: artes y ciencias, áreas diversas, y combinado con formación técnica y vocacional (programas de dos años).

- Colleges de associate's que entregan diplomas de pregrado predominantemente en programas de dos años, de tal suerte que los graduados de programas de cuatro años no alcanzan al 10\% del total de graduados de pregrado. Esta categoría tiene 14 subgrupos según si el college es público o privado, con o sin fines de lucro, su tamaño, su número de campus, si es urbano, suburbano o rural, y si pertenece a una universidad o college mayor.

- Instituciones especializadas (en Teología, Derecho, Medicina, Ingeniería, Administración, etc.).

- Colleges tribales.

Hay que decir que lo que la clasificación Carnegie ha ganado en matices, complejidad y sutileza, lo ha perdido en simplicidad y facilidad de uso. De este modo, la comprensión cabal de la fórmula usada para clasificar universidades doctorales en alguno de los tres grupos que se distinguen en esa clase exige ahora conocimientos no triviales de estadística.

Con todo, la "clasificación básica" es una buena ilustración de una tipología basada principalmente en funciones y resultados. Nótese que no se miden los alumnos matriculados o el número de grados ofrecidos, sino los grados académicos otorgados.

A propósito de la selectividad en el ingreso de estudiantes, la comisión Carnegie decidió eliminarla en 2000 como criterio de clasificación -la usaba al interior de la clase de los colleges de pregrado- en atención a que el criterio se solía emplear (erradamente a juicio de la comisión) como equivalente a "calidad", y a que se refería exclusivamente a un insumo, sin entregar información acerca de lo que la institución hacía con éste, entre otras razones ${ }^{14}$.

14 The Carnegie Foundation for the Advancement of Teaching. (2001). The Carnegie Classification of Institutions of Higher Education, 2000 Edition. Menlo Park, CA: The Carnegie Foundation for the Advancement of Teaching, pp. 15-16. 


\section{Conclusiones}

A mi juicio, sería más informativa para los usuarios del sistema, los investigadores, los observadores internacionales y las propias instituciones, una clasificación que ponga el acento en las funciones y resultados de la universidad, porque me parece que estos aspectos tienen más que ver con la identidad y la misión real de una universidad que las características de los alumnos que ingresan, o el tamaño, o la naturaleza jurídica, o la misión declarada, o la ubicación geográfica de la institución. Enfatizar las funciones y resultados significa - por ejemplo, en materia de áreas del conocimiento que la universidad cubre- no clasificar sobre la base de las áreas en que la universidad ofrece programas, sino según la distribución por áreas de los grados y títulos que otorga. Significa también considerar el número de diplomas de posgrado que emite, las publicaciones indexadas que genera o la cantidad de alumnos internacionales que recibe por intercambio.

Es cierto que una aplicación estricta de muchos de estos indicadores discriminaría poco, en el sentido de que habría un puñado de universidades en la clase de instituciones orientadas a la investigación, mientras que todas las demás se amontonarían indiferenciadamente en la clase de universidades docentes. Pero es aquí donde la aplicación jerárquica de criterios de clasificación ayuda: una vez separadas las instituciones con investigación significativa de las que no la poseen, se pueden desplegar criterios secundarios de diferenciación al interior del segundo grupo, relacionados, por ejemplo, con las características de la docencia de pregrado y magíster, tal como lo hace la clasificación Carnegie.

Por supuesto, optar por las funciones de la universidad como criterio principal de clasificación es una cuestión de objetivos y, en cierta medida, también de gustos; por otra parte, sin duda que una clasificación basada en funciones tendría también limitaciones y quedaría expuesta a justificadas críticas. Clasificar universidades no es tarea fácil, no sólo por la pluralidad de puntos de vista para 


\section{clasificar y la complejidad técnica del proceso de convertir criterios en variables y de medir esas variables más o menos objetivamente, sino por la inevitable asociación que se hace entre clasificación y ranking.}

Tipología de instituciones universitarias*

\begin{tabular}{|c|c|}
\hline VARIABLE & CRITERIOS DE CLASIFICACIÓN \\
\hline $\begin{array}{l}\text { TIPO DE } \\
\text { UNIVERSIDAD }\end{array}$ & $\begin{array}{l}\text { Pu-T: Pública tradicional } \\
\text { Pu-D: Pública derivada } \\
\text { PR: Privada }\end{array}$ \\
\hline TAMAÑO & $\begin{array}{l}\text { G: Grande ( } 15 \text { mil alumnos o más) } \\
\text { MG: Mediano-grande ( } 10 \text { mil alumnos a } 14.999 \text { alumnos) } \\
\text { M: Mediano (5.000 a } 9.999 \text { alumnos) } \\
\text { MP: Mediano-Pequeño ( } 1.000 \text { a } 4.999 \text { alumnos) } \\
\text { P: Pequeño (999 alumnos o menos) }\end{array}$ \\
\hline COBERTURA & $\begin{array}{l}\text { COM: Completa ( } 10 \text { áreas) } \\
\text { A: Amplia ( } 7,8 \text { o m } 9 \text { áreas) } \\
\text { CON: Concentrada ( } 4,506 \text { áreas) } \\
\text { E: Especializada ( } 1,2 \text { o } 3 \text { áreas o más de c/ } 50 \% \text { o más de la matrícula en una sola área) }\end{array}$ \\
\hline SELECTIVIDAD & $\begin{array}{l}\text { SA: Súper Alta (índice de } 80 \% \text { o más) } \\
\text { A: Alta (índice de } 60 \% \text { a } 79 \% \text { ) } \\
\text { M: Media (de } 40 \% \text { a } 59 \% \text { ) } \\
\text { MB: Media Baja (índice de } 20 \% \text { a } 39 \% \text { ) } \\
\text { B: Baja (índice de } 5 \% \text { a } 19 \% \text { ) } \\
\text { NS: No Selectiva (índice inferior a } 5 \% \text { ) }\end{array}$ \\
\hline $\begin{array}{l}\text { ÍNDICE DE } \\
\text { DENSIDAD DE } \\
\text { CONOCIMIENTO } \\
\text { (IDC) }\end{array}$ & $\begin{array}{l}\text { Combina } 5 \text { indicadores } \\
\text { Idcisi-N }{ }^{\circ} \text { de publicaciones. Sí de cada universidad/total publicaciones ISI durante } \\
\text { años } 2001 \text { a } 2003 \\
\text { Idcproy-N } N^{\circ} \text { proyectos de investigación de cada universidad en ejecución año } 2004 \\
\text { Idcmpost - matrícula de posgrado de cada universidad/matrícula total posgrado } \\
\text { universitaria año } 2003 \\
\text { Idcconap - } \mathrm{N}^{\circ} \text { programas acreditados o reactivados de doctorado y maestría por } \\
\text { cada universidad / total de dichos programas, noviembre } 2004 \\
\text { Idccha - No titulados por universidad año } 2000 / \text { total titulados universidades año } 2002 \\
\text { Fórmula de cálculo : IDC=0.2* idcisi + 0.15* idcmpost + 0.1 idcconap + 0.4* idcfcha } \\
\text { * Incluye proyectos FONDECYT, FONDEF, FONDAP, Programa Nacional Becas } \\
\text { Postgrado, Fondos regionales y de divulgación C y T de CONICYT, FIA, MILENIO } \\
\text { MA: Muy Alta densidad (IDC de 10 o más) } \\
\text { A: Alta densidad (IDC de } 5 \text { a 9,99) } \\
\text { M: Media densidad (IDC de } 2 \text { a 4,99) } \\
\text { B: Baja densidad (índice de } 1 \text { a 1,99) } \\
\text { MB: Muy Baja densidad (índice menos de } 1 \text { ) }\end{array}$ \\
\hline
\end{tabular}

* Estas tablas se incluyen en el informe "Guiar el mercado. Información sobre la educación superior chilena" de los autores José Joaquín Brunner, Gregory Elacqua, Anthony Tillet y colaboradores. 


\begin{tabular}{l|l}
\hline CARÁCTER & PP: PRINCIPIOS PLURALISTAS. Correspondientes a aquellas instituciones que \\
DE LA MISIÓN & enfatizan valores de pluralidad, diversidad, participación y democracia. \\
DECLARADA* & VR: VOCACIÓN REGIONAL: Corresponde a aquellas instituciones que declaran \\
su contribución al desarrollo de la región en la que tiene su sede principal y se \\
identifican como factor de desarrollo de la misma. \\
RP: RELIGIOSA PASTORAL: Corresponde a aquellas instituciones que declaran \\
explícitamente su vínculo con alguna confesión religiosa y definen su misión en \\
torno a los valores y la visión que ella sustenta. \\
BEC: BASE ÉTICO-CRISTIANA: Corresponde a aquellas instituciones que enfatizan \\
su compromiso con una formación con base ética fundada en los valores del \\
humanismo cristiano.
\end{tabular}

* Cada tipo de misión ha sido definido sobre la base del aspecto mas destacado por la propia institución en sus enunciados. Esto no excluye la posibilidad de que en cada caso estén presentes elementos secundarios; por ejemplo, una misión institucional puede ser religioso-pastoral pero, al mismo tiempo, incluir elementos de énfasis empresarial o de principios pluralistas. En algunos casos resulta difícil conciliar la misión declarada con la que el público reconoce como la visión o postura dominante de una institución. En estos casos se ha optado por recoger los elementos enunciados, at face value.

Sobre la base de estos criterios, pueden distinguirse las siguientes ocho categorías de universidades en el sistema institucional chileno:

\begin{tabular}{l|l}
\hline I. & Universidades con liderazgo académico internacionalizado \\
\hline II. & Universidades públicas con proyección nacional \\
\hline III. & Universidades privadas con liderazgo selectivo \\
\hline IV. & Universidades estatales metropolitanas especializadas \\
\hline V. & Universidades regionales menos selectivas \\
\hline VI. & Universidades privadas con selectividad media baja \\
\hline VII. & Universidades públicas y privadas con baja selectividad \\
\hline VIII. & Universidades privadas no selectivas \\
\hline
\end{tabular}

Recibido: 24 de agosto de 2006

Aprobado: 31 de agosto de 2006 